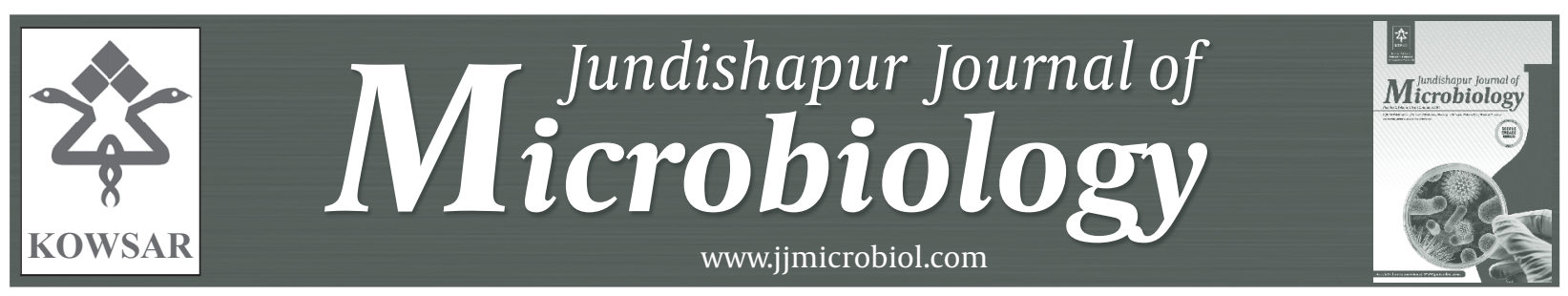

\title{
Assessment of Carbapenem Susceptibility and Multidrug-Resistance in Pseudomonas aeruginosa Burn Isolates in Tehran
}

\author{
Somayeh Moazami-Goudarzi ${ }^{1}$, Fereshteh Eftekhar ${ }^{{ }^{*}}$ \\ ${ }^{1}$ Departement of Microbiology, Faculty of Biological Sciences, Shahid Beheshti University, Tehran, IR Iran \\ * Corresponding author: Fereshteh Eftekhar, Department of Microbiology, Faculty of Biological Sciences, Shahid Beheshti University, G.C, Evin, Tehran, IR \\ Iran.Tel.: +98-2129903208, Fax: +98-2122431664, E-mail: f-eftekhar@sbu.ac.ir
}

\begin{abstract}
A B S T R A C T
Background: Pseudomonas aeruginosais an opportunistic pathogen which causes severe, acute and chronic nosocomial infections. These infections are difficult to eradicate since the organisms are usually multidrug-resistant. Carbapenems are considered as the most effective drugs against these isolates. However, recent emergence of carbapenem-resistant $P$. aeruginosa has become a major healthcare problem.

Objectives: The present study was conducted to determine the antibiotic susceptibility of $P$. aeruginosa burn isolates to 13 antibiotics including imipemen and meropenem.

Materials and Methods: One hundred and thirty three P. aeruginosa burn isolates were collected from Shahid Motahari Burn Hospital between July and December 2011. The majority of the isolates were from wounds (88.7\%), followed by 5.26\% from blood, $4.15 \%$ from subclavian catheters and $1.5 \%$ from urine. The antibiotic susceptibility profiles were studied by the agar disc diffusion.

Results: The results showed $99.2 \%$ resistance to carbenicillin, $98.4 \%$ to ticarcillin, $96.2 \%$ to ciprofloxacin, $95.4 \%$ to co-trimoxazole, $94.7 \%$ to imipenem and meropenem, $93.9 \%$ to piperacillin, $93.2 \%$ to azetronam, $92.4 \%$ to tobramycin, $91.7 \%$ to cefepime, $89.4 \%$ to amikacin and ceftazidime, and finally $87.2 \%$ to piperacillin-tazobactam. Overall, $100 \%$ of the isolates showed multidrug-resistance (resistance to $\geq 3$ classes of antibiotics) including theimipenem- resistant isolates.

Conclusions: The high rate of multidrug-resistance is alarming and it is crucial to screen for carbapenem resistance prior to - antibiotic therapy.
\end{abstract}

Keywords: Pseudomonas aeruginosa; Antibiotic Susceptibility; Carbapenems

Copyright @ 2013, Kowsar Corp.; Published by Kowsar Corp.

Article type: Research Article; Received: 03 Apr 2012, Revised: 07 May 2012, Accepted: 07 Jun 2012; DOI: 10.5812/jjm.5036

Implication for health policy/practice/research/medical education:

Suitable protocols are needed for appropriate use of antibiotics for treatment of multidrug resistant bacterial infections as well as avoidingthe spread of resistance genes among bacteria.

Please cite this paper as:

Moazami-Goudarzi S, Eftekhar F. Assessment of Carbapenem Susceptibility and Multidrug-Resistance in Pseudomonas aeruginosa Burn Isolates in Tehran. Jundishapur J Microbiol. 2013;6(2):162-165. DOI:10.5812/jjm.5036

Copyright@ 2013, Ahvaz Jundishapur University of Medical Sciences; Published by Kowsar Corp.

This is an Open Access article distributed under the terms of the Creative Commons Attribution License(http://creativecommons.org/licenses/by/3.0), which permits unrestricted use, distribution, and reproduction in any medium, provided the original work is properly cited. 


\section{Background}

Pseudomonas aeruginosa is the most common cause of $10 \%$ of all hospital-acquired infections (1). Infections occur particularly in immunocompromised hosts suffering from respiratory diseases, cancer and burns as well ascystic fibrosis with a significant rate of morbidity and mortality $(2,3)$. Burn injuries resulting in open and large wounds, including those with necrotic tissues, make these patients more susceptible to infection with $P$. aeruginosa $(4,5)$. Treatment of infections caused by $P$. aeruginosa is frequently complicated due to the limited susceptibility to antimicrobial agents and the emergence of antibiotic resistance during therapy. As a result, due to the severe adverse outcomes, the mortality rate among infected patients is likely to reach up to $40-50 \%(2,5)$.

The Emergence of multidrug-resistant (MDR) strains in burns units, particularly in economically underdeveloped and developing countries, has become a major problemin the control of infections $(6,7)$. Multidrug-resistance, caused by a variety of resistance mechanisms, leaves few alternatives for treatment of some patients (4). Carbapenems are the selective drugs for treatment of MDR isolates $(2,6)$. However, the increasing frequency of carbapenem-resistant $P$. aeruginosa has recently become a worldwide serious concern. The prevalence of imipenem-resistant $P$. aeruginosain Tehran has been reported to be within the range of $16 \%$ to $100 \%(8,9)$. In fact, in the past two years, the rate of imipenem-resistant $P$. aeruginosa has increased from 69\% to $97.5 \%$ in Shahid Motahari Burn Hospital $(9,10)$.

\section{Objectives}

Multidrug-resistant $P$. aeruginosa is the most prevalent bacterial isolate among the burn patients. The objective of the study was to determine the antibiotic susceptibility profiles of $P$. aeruginosa burn isolates in Shahid Motahari Burn Hospital in Tehran.

\section{Materials and Methods}

\subsection{Bacterial Isolates}

One hundred and thirty three $P$. aeruginosa burn isolates were collected from Shahid Motahari Burn Hospital between July and December 2011. The majority of bacterial isolates were recovered from wounds (88.72\%), followed by blood (5.26\%), cvp (subclavian catheters) lines (4.51\%) andurine (1.5\%). Two thirds of the specimens were from male patients and the rest were from females. All isolates were identified as P. aeruginosa by the standard microbiological tests such as Gram stain, oxidase test, growth on MacConkey agar (Liofilchem, Italy), oxidationfermentation test, growth and fermentation on triple sugar iron agar (QBBL, UK), growth at $42^{\circ} \mathrm{C}$, and pigment formation (11). The isolates were maintained at $-20 \mathrm{oC}$ in brain -heart infusion broth (Oxoid, UK) containing 10\% dimethyl sulfoxide (v/v).

\subsection{Antimicrobial Susceptibility}

The Antimicrobial susceptibility profiles of the isolates were determined by the agar disc diffusion method according to the recommendations made by the Clinical and Laboratory Standards Institute (CLSI) (12). The following antimicrobial discs (MAST Diagnostics, Merseyside, UK) were used: imipenem (10 $\mu \mathrm{g})$, meropenem (30 $\mu \mathrm{g})$, aztreonam $(30 \mu \mathrm{g})$, cefepime $(30 \mu \mathrm{g})$, ceftazidime $(30 \mu \mathrm{g})$, amikacin $(30 \mu \mathrm{g})$, ciprofloxacin $(5 \mu \mathrm{g})$, piperacillin (100 $\mu \mathrm{g})$, piperacillin/tazobactam $(110 \mu \mathrm{g})$, carbenicillin (100 $\mu \mathrm{g})$, ticarcillin $(75 \mu \mathrm{g})$, co-trimoxazole $(25 \mu \mathrm{g})$ and tobramycin $(10 \mu \mathrm{g})$. P. aeruginosa ATCC 27853 was used as the control in each run of antimicrobial susceptibility tests which were repeated at least 3 times and the average of inhibition zones was reported.

\section{Results}

The antibiotic susceptibility results showed $99.2 \%$ resistance to carbenicillin, $98.4 \%$ to ticarcillin, $96.2 \%$ to ciprofloxacin, $95.4 \%$ to co-trimoxazole, $94.7 \%$ to imipenem and meropenem, $93.9 \%$ to piperacillin, $93.2 \%$ to azetronam, 92.4\% to tobramycin, $91.7 \%$ to cefepime, $89.4 \%$ to amikacin and ceftazidime, and $87.2 \%$ to piperacillin-tazobactam ( Figure 1 ). All isolates were MDR (resistant to at least 3 different classes of antibiotics).

Figure 1. Antibiotic Susceptibility of P. aeruginosa Burn Isolates.

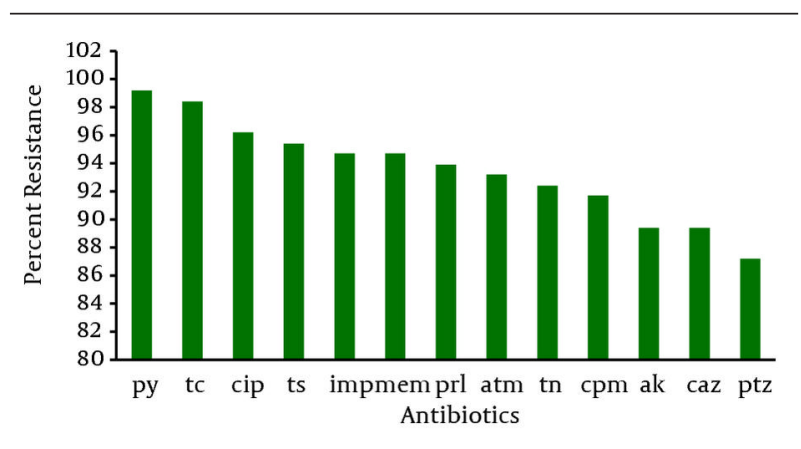

Py: carbenicillin, Tc: ticarcillin, Cip: ciprofloxacin, Ts: co-trimoxazole, Imp: imipenem, Mem: meropenem, Prl: piperacillin, Atm: aztreonam, Tn: tobramycin,Cpm: cefepime, Ak: amikacin, Caz: ceftazidime and Ptz: piperacillin/tazobactam.

Among the imipenem-resistant $P$. aeruginosa, there was $99.2 \%$ resistance to carbenicillin, $98.4 \%$ to ticarcillin, $96.8 \%$ to ciprofloxacin and azetronam, 96\% to meropenem, $95.3 \%$ to co-trimoxazole, $94.5 \%$ to tobramycin, $93.7 \%$ to cefepime and piperacillin, $91.4 \%$ to amikacin, $89.4 \%$ to ceftazidime and $87.5 \%$ to piperacillin-tazobactam (Figure 2 ). Since there was an overall high rate of imipenem resistance (94.7\%), no statistical analysis could be performed to compare the susceptibility profiles of imipenem-resistant 
strains with the susceptible isolates. However, resistance to ceftazidime, tobramycin, meropenem, cefepime and ciprofloxacin was slightly higher in imipenem-resistant strains ( Figure 2 ), there was no relationship between antibiotic resistance and the source of the specimen.

Figure 2. Comparsion of Antibiotic Susceptibility Between Imipenem Resistant and All of $P$. aeruginosa Isolates.

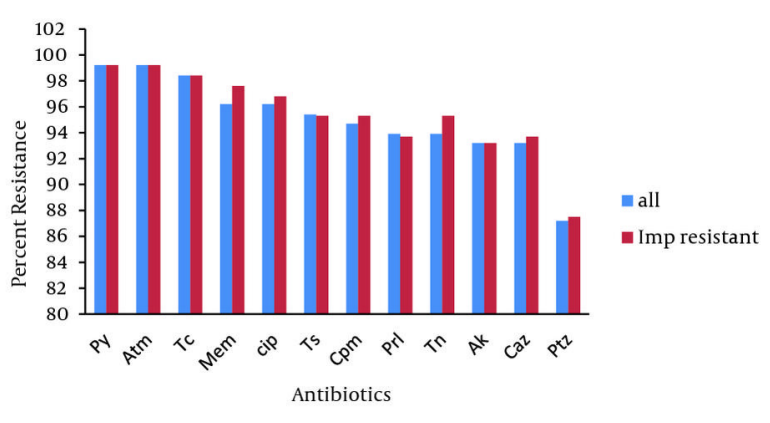

Py: carbenicillin, Atm: aztreonam,Tc:ticarcillin, Mem: meropenem, Cip ciprofloxacin, Ts: co-trimoxazole,Cpm: cefepime, Prl: piperacillin, Tn: tobramycin,Ak: amikacin, Caz: ceftazidime and Ptz: piperacillin/tazobac tam.

\section{Discussion}

Over the past 20 years, $P$. aeruginosa has been repeatedly recognized as the most prevalent organism which causes infection in burn centers in Tehran $(5,13,14)$. Unfortunately, the organism appears to be resistant to almost all antimicrobial agents creating a great problem in clinical settings. In addition, the more recent emergence of carbapenem resistance in $P$. aeruginosa has limited the therapeutic options.

Multidrug-resistant $P$. aeruginosa is often the cause of outbreak of diseases in the burn units $(5,10)$. Different rates of MDR have been reported for $P$. aeruginosa burn isolates in various cities of $\operatorname{Iran}(5,10,13)$. The reports from Shahid Motahari Burn Hospital in Tehran have varied considerably from 2007 to 2011 in both MDR and carbapenem resistance. Salimi et al. found 16\% imipenem resistance and $42 \%$ MDR in P. aeruginosa isolates collected in 2008 from the intensive care burn patients (14). Saderi et al. reported that $69 \%$ of $P$. aeurginosa burn isolates in 2008 were multidrug-resistant and MDR was more prevalent in the imipenem- resistant strains compared to the imipenem susceptible isolates (87\% vs. 29\%) (10). In 2007 , Ranjbar et al. reported that all P. aeruginosa burn isolates from Shahid Motahari Burn Hospital were MDR of which, 97.5\% were resistant to imipenem (9).

We found that $100 \%$ of P. aeurginosa isolates from Shahid Motahari Burn Hospital were MDR of which 94.7\% were resistant to both imipenem and meropenem. The main reasons for various rates of drug resistance in such a short period of time from the same hospital could be the use of different antibiotic regimes, presence of different persistent strains in hospitals and the quality of hygiene in different environments. Similarly, according to other studies in Iran, various degrees of imipenem resistance in $P$. aeruginosa have been reported. Beheshti and Zia reported $61.1 \%$ imipenem resistance in $P$. aeruginosa burn isolates from Imam Mousa Kazem Burn Center in Esfahan in 2011 (13). Haghi et al. reported $100 \%$ multidrug and imipenem resistance in P. aeruginosa burn isolates in Orumieh (13). Jamali and colleagues reported $61.8 \%$ imipenem resistance in the burn isolates of $P$. aeruginosa in 2009 (15). Studies in other countries have also shown different rates of imipenem resistance in $P$. aeruginosa burn isolates. Similar to these results, Shahid et al. found $100 \%$ MDR in P. aeurginosa burn isolates in India in 2003, (16). In 2004, Ozkurt et al. reported 69.92\% imipenem resistance in $P$. aeruginosa burn isolates in Turkey (17). In a study conducted between 1996 and 1998 in Korea, 52\% of P. aeruginosa were resistant to imipenem(18). In another study performed in Karachi, Pakistan in 2003, imipenem resistance in P. aeruginosa was $32.7 \%$ (19).

Antibiotic resistance in P. aeruginosa may be mediatedvia several distinct mechanisms including $\beta$-lactamase production, efflux pumps, modification of site-targeted drugs or outer membranes $(4,20)$. MDR is usually the result of a combination of different mechanisms in a single isolate or the action of a single potent resistance mechanism (20). The Increase in antibiotic resistance is mostly due to extensive use of antibiotics such as ciprofloxacin, $\beta$-lactams and aminoglycosides in the burn centers as well as non-availability and high costs of other effective drugs.

We believe that it is important to conduct surveillance programs for appropriate empirical therapy and the practices of infection control. Meanwhile, it is necessary for health care practitioners and policy makers to address this problem by implementing suitable protocols foruse of antibiotics not only to find strategies for treating such difficult infections, but also to avoid spreading of the resistance genes among bacteria.

\section{Acknowledgements}

The authors wish to thank Shahid Beheshti University Research Council for providing a special grant to finance this research and Shahid Motahari Burn Hospital in Tehran for supplying the bacterial isolates.

\section{Financial Disclosure}

None declared.

\section{Funding/Support}

This research was financially supported by Shahid Beheshti University Research Council. 


\section{Authors' Contribution}

None declared.

\section{References}

1. National Nosocomial Infections Surveillance (NNIS) System Report, data summary from January 1992 through June 2004, issued October 2004.National Nosocomial Infections Surveillance (NNIS) System Report, data summary from January 1992 through June 2004, issued October 2004. Am J Infect Control. 2004;32(8):470-85.

2. Rossolini GM, Mantengoli E. Treatment and control of severe infections caused by multiresistant Pseudomonas aeruginosa. Clin Microbiol Infect. 2005;11 Suppl 4:17-32.

3. Sadikot RT, Blackwell TS, Christman JW, Prince AS. Pathogenhost interactions in Pseudomonas aeruginosa pneumonia. Am J Respir Crit Care Med. 2005;171(11):1209-23.

4. Lister PD, Wolter DJ, Hanson ND. Antibacterial-resistant Pseudomonas aeruginosa: clinical impact and complex regulation of chromosomally encoded resistance mechanisms. Clin Microbiol Rev. 2009;22(4):582-610.

5. Rastegar Lari AR, Alaghehbandan R, Akhlaghi L. Burn wound infections and antimicrobial resistance in tehran, iran: an increasing problem. Ann Burns Fire Disasters. 2005;18(2):68-73.

6. Japoni A, Farshad S, Alborzi A. Pseudomonas aeruginosa: Burn Infection, Treatment and Antibacterial Resistance. Iran Red Crescent Med J. 2009;11(3):244-53.

7. Moniri R, Mosayebi Z, Movahedian AH, Mousavi GA, GA M. Emergence of multi-drug-resistant Pseudomonas aeruginosa isolates in neonatal septicemia. J Infect Dis Antimicrob Agents. 2005;22(2):39-44.

8. Bayat M, Zia M, Haghi M, Hemmatyar G, Toghyani M. Antibiotic resistance pattern of Escherichia coli, Staphylococcus aureus and Pseudomonas aeruginosa isolated from burnt patients in Urmia, Iran. Afr J Microbiol Res. 2011;5(9):996-1000.
9. Ranjbar R, Owlia P, Saderi H, Mansouri S, Jonaidi-Jafari N, Izadi M, et al. Characterization of Pseudomonas aeruginosa strains isolated from burned patients hospitalized in a major burn center in Tehran, Iran. Acta Med Iran. 2011;49(10):675-9.

10. Saderi H, Lotfalipour H, Owlia P, Salimi H. Detection of Metallo$\beta$-Lactamase Producing Pseudomonas aeruginosa Isolated From Burn Patients in Tehran, Iran. Lab Medicine. 2010;41(10):609-12.

11. Forbes BA, Sahm D, Weissfeld A. Diagnostic microbiology. Bailey \& Scott1s Diagnostic Microbiology. 1966;11.

12. Wikler MA. Performance standards for antimicrobial susceptibility testing: Sixteenth informational supplement. Clinical and Laboratory Standards Institute. 2006.

13. Beheshti S, Zia M. Bacteriology of burns and antibiogram in an Iranian burn care center. Afr J Pharm Pharmacol. 2011;5(4):538-41.

14. Salimi H, Yakhchali B, Owlia P, Lari AR. Molecular Epidemiology and Drug Susceptibility of Pseudomonas aeruginosa Strains Isolated From Burn Patients. Lab Medicine. 2010;41(9):540-4.

15. Jamali S, Bahar M, Houshmand S. J Microbiol Knowledge. 2009; p.

16. Shahid M, Malik A, Sheeba . Multidrug-resistant Pseudomonas aeruginosa strains harbouring R-plasmids and AmpC beta-lactamases isolated from hospitalised burn patients in a tertiary care hospital of North India. FEMS Microbiol Lett. 2003;228(2):181-6.

17. Ozkurt Z, Ertek M, Erol S, Altoparlak U, Akcay MN. The risk factors for acquisition of imipenem-resistant Pseudomonas aeruginosa in the burn unit. Burns. 2005;31(7):870-3.

18. Song W, Lee KM, Kang HJ, Shin DH, Kim DK. Microbiologic aspects of predominant bacteria isolated from the burn patients in Korea. Burns. 2001;27(2):136-9.

19. Naqvi ZA, Hashmi K, Rizwan QM, Kharal SA. Multidrug resistant Pseudomonas aeruginosa: a nosocomial infection threat in burn patients. Pakistan J Pharma. 2005;22(2):9-15.

20. Tavajjohi Z, Moniri R, Khorshidi A. Detection and characterization of resistance and extended spectrum $\beta$-lactamase producing (ESBL) Pseudomonas aeruginosa isolates in a teaching hospital. Afr J Microbiol Res. 2011;5(20):3223-28. 